Available online at GSC Online Press Directory

GSC Biological and Pharmaceutical Sciences

e-ISSN: 2581-3250, CODEN (USA): GBPSC2

Journal homepage: https://www.gsconlinepress.com/journals/gscbps

(RESEARCH ARTICLE)

\title{
Recent pollen rain analysis in Akoko environment as indicator of the vegetation of Ondo State, Nigeria
}

\author{
Essien Benjamin Christopher * and Ige Olugbenga Ebenezer \\ Department of Plant Science and Biotechnology, Faculty of Science, Adekunle Ajasin University, Akungba-Akoko, Ondo \\ State, Nigeria.
}

Publication history: Received on 23 May 2020; revised on 09 July 2020; accepted on 11 July 2020

Article DOI: https://doi.org/10.30574/gscbps.2020.12.1.0149

\begin{abstract}
The study of Atmospheric Pollen Content (APC) in Akoko environment provides standard baseline information on environmental change, vegetation type, species composition and their utilization for sustainable development. This study was carried out in twenty randomly selected locations to examine the temporal and spatial relationship between the atmospheric pollen content and their vegetation. The palynomorphs were collected with Modified Tauber Sampler using simple random sampling technique and analyzed palynologically. Results showed 182 pollen types belonging to 76 angiospermic plant families were encountered. Three (3) were identified to family level, 57 to species level, 121 to generic level and one (1) unidentified. The predominant pollen types belong to the family Poaceae, Elaeis guineensis, Alchornea cordifolia, Aspilia africana, Casuarina equisetiifolia and Tridax procumbens. The presence of Empetrum nigrum, Linnea borealis, Stereospermum kunthianum and Vitellaria paradoxa pollen indicates evidence of long distance transport. Pollen of exotic or introduced plants was part of the atmospheric palynomorphs recorded as evidence of human impact. Airborne pollen grains were most abundant between September and January (late rainy season to early dry season). Rainfall and relative humidity had negative effect on pollen concentration. Percentage composition of pollen grains abundance showed that indicators of the Savanna taxa were the highest pollen contributors (55.74\%) followed by Open Forest (22.72\%), Human Impact (14.32\%) and Lowland Rainforest (7.21\%) taxa respectively. The various ecological indicators species identified confirmed their origin as coming from the Forest- Savanna ecological zone that is anthropogenically disturbed, which is characteristic of Akoko environment. Pollen assemblages confirmed the vegetation of the study area to be Tropical Rainforest vegetation type despite high level of anthropogenic activities on the environment.
\end{abstract}

Keywords: Akoko Environment; Airborne; Pollen rain; Ondo State; Vegetation

\section{Introduction}

The vegetation of an area is an integral and basic component of the ecosystem and is sensitive to changes in the ecosystem [1]. Consequently, vegetation changes are themselves a response to and a reflection of variation in one or more of the factors of the environment, particularly climate. According to Sowunmi [2], a close relationship exists between vegetation and the rest of the environment, particularly climate and soil. Thus, the flora of an area provides a good reflection of the major climatic regime of the area. The influence of climate on other components of the environment is so great that every other climatic zone has its own characteristic vegetation type.

Plants are therefore among the best indicators of the environment especially of the climate, soil and fauna [3]. Certain individual or assemblages of plants are known to be characteristic of specific ecological zone and the occurrence of the fossils of such ecological indicator species in sediments is considered a reflection of contemporary ecological conditions [4]. For example, the tropical rainforest is characterized by broad-leaved species, the savanna characterized by grasses,

\footnotetext{
* Corresponding author: Essien Benjamin Christopher
} 
the desert by ephermeral and sclerophyllous plants and the cold regions of the world characterized by evergreen conifers [5].

The study of vegetation and the way in which it has been altered and developed in the course of time indicates past changes that have occurred in our terrestrial environment [6]. Variations in climate and in the intensity of human activities in historic and prehistoric times have made their mark upon vegetation, and the plants themselves have left a record of these changes in the form of vast quantities of pollen grains which have survived in contemporary sediments [7].

The most common plant remains in Quaternary deposits are the pollen grains of flowering plants (Spermatophytes) and the spores of ferns (Pteridophytes) or mosses (Bryophytes); all of which are not visible to the naked eyes. The examination of pollen grains and spores do not only provide qualitative information on the plant present, but also quantitative information on their abundance. Thus, conclusion can be drawn about former vegetation conditions [8], [9].

The relevance of atmospheric pollen content to the vegetation of a region is related to the palynomorphs produced in situ and those supplied from the surrounding zones [10], [11]. Pollen and spores are generally produced in excess of the biological needs of the plants. Established observations have shown that anemophilous plants release large quantities of pollen into the air, while entomophilous taxa release smaller amounts [12]. The behavioural patterns lead to differences in the quantity of pollen and spores of various taxa available in the atmosphere, and which of them can be trapped during sampling [13].

Some factors that aid in the dispersal are the size, shape and density of the pollen and spores, the position of the parent plants (that produce them) in the vegetation and the prevailing climatic conditions [14]. The distinctive surface sculptures of pollen grains aid in the identification of their parent plants to at least family level [15]. Their other qualities such as small size, abundance and high resistance to degradation have made them an invaluable evidence of vegetation as well as environmental changes [16]. Because of the transitional location of Akoko division, Ondo State, Nigeria between the tropical rainforest in the South and the true savanna in the North; the area receives particles moving from the South to the Northern part of Nigeria and vice versa. Equally, the movements of the South-West Monsoon and NorthEast Trade winds across the latitudinal zones of Nigeria carrying along with them large quantities of particles which are deposited along their routes depending on the strength of the winds during the major seasons of the year. The aim of this study is to determine the taxa of pollen types present in the atmosphere of the study environment and the relationship between airborne pollen assemblages with its vegetation.

\section{Material and methods}

\subsection{Location of the study area}

Akoko division of Ondo State is located at Latitude $7^{0} 31^{\prime} 28.13^{\prime \prime} \mathrm{N}$ and Longitude $5^{0} 46^{\prime} 9.25^{\prime \prime} \mathrm{E}$ and an elevation of 526.88 $\mathrm{m}$ above sea level. It is comprises of a number of smaller towns, villages and homestead, whose inhabitants have in numerous ways left their impact on the environment.

\subsection{Geology}

There are two distinct geological regions in Ondo State. First, is the region of sedimentary rocks in the south, and secondly, the region of Precambrian Basement Complex rocks in the north. Some few kilometres north of Aaye occurs the basement complex sedimentary rocks boundary. The sedimentary rocks are mainly of the post-Cretaceous sediments and the Cretaceous Abeokuta Formation. The basement complex is mainly of the medium grained gneisses. These are strongly foliated rocks frequently occurring as outcrops. On the surface of these outcrops, severely contorted, alternating bands of dark and light coloured minerals can be seen. These bands of light coloured minerals are essentially feldspar and quartz, while the dark-coloured bands contain abundant biotic mica. A small proportion of the State, especially to the northeast, overlies the coarse grained granites and gneisses, which are poor in dark ferromagnesian minerals.

\subsection{Climate}

In the south, the mean monthly temperature is $27^{\circ} \mathrm{C}$, with a mean monthly range of $2^{\circ} \mathrm{C}$, while mean relative humidity is over seventy five percent. However, in the northern part of the State, the mean monthly temperature and its range are about $30^{\circ} \mathrm{C}$ and $6^{\circ} \mathrm{C}$ respectively. The mean monthly relative humidity is less than seventy percent. In the south, rain 
falls throughout the year, but the three months of November, December and January may be relatively dry. The mean annual total rainfall exceeds 2000 millimetres. However, in the north, there is a marked dry season from November to March when little or no rain falls. The total annual rainfall in the north, therefore, drops considerably to about 1800 millimetres.

\subsection{Vegetation}

The natural vegetation of Ondo State is the Tropical Rainforest type, composed of many varieties of hardwood timber such as Milicia excelsa, Antiaris africana, Terminalia superba and Symphonia globulifera. In the northern districts, the vegetation consists of woody savanna featuring such tree species as Blighia sapida and Parkia biglobosa. The swamp flats are the domain of the fresh water swamp forests in the interior and units of mangrove vegetation near the coast. The sand ridges are characterized by savanna and stunted rain forests taxa. Over most part of the State, the natural vegetation has been very much degraded as a result of human activities, the chief of which is based on the rotation of bush fallow system and lumbering. As a result of anthropogenic activities, Government has restricted the original forest to forest reserves [5], [17].

An important aspect of the vegetation of the Ondo State is the prevalence of tree crops. The major tree crops include Theobroma cocao, Cola nitida, Coffee arabica, Hevea brasiliensis, Elaeis guineensis and Citrus sp.; cocoa being the most prevalent. It is also important to note that rubber and oil palms have been cultivated in large plantations in Odigbo, Okitipupa and Irele Local Government Areas. Trees that are not native have also been introduced as forest plantations. These exotic species have been used to re-vegetate large portions of harvested old forest reserves in Omo and Owo. They include mainly Tectona grandis (teak) and Gmelina arborea (pulp wood). Map of the study area are shown below:

a.

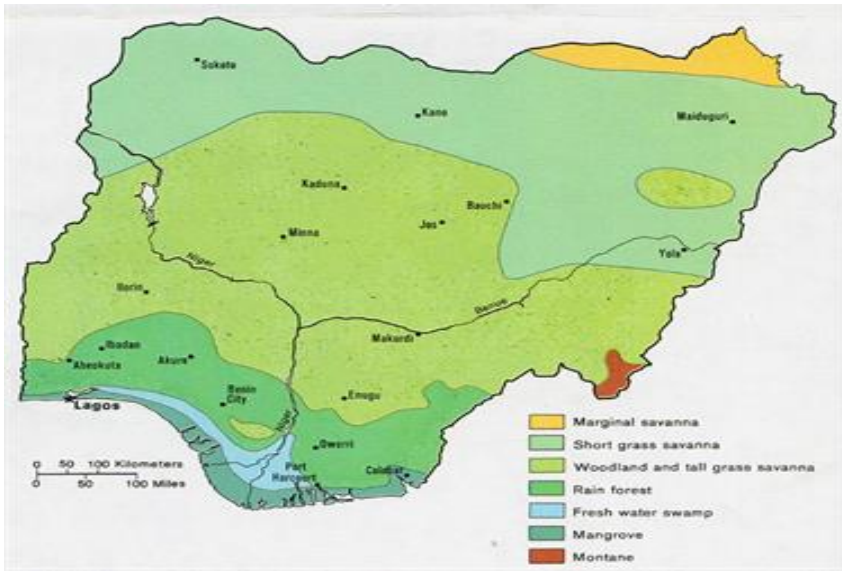

b.

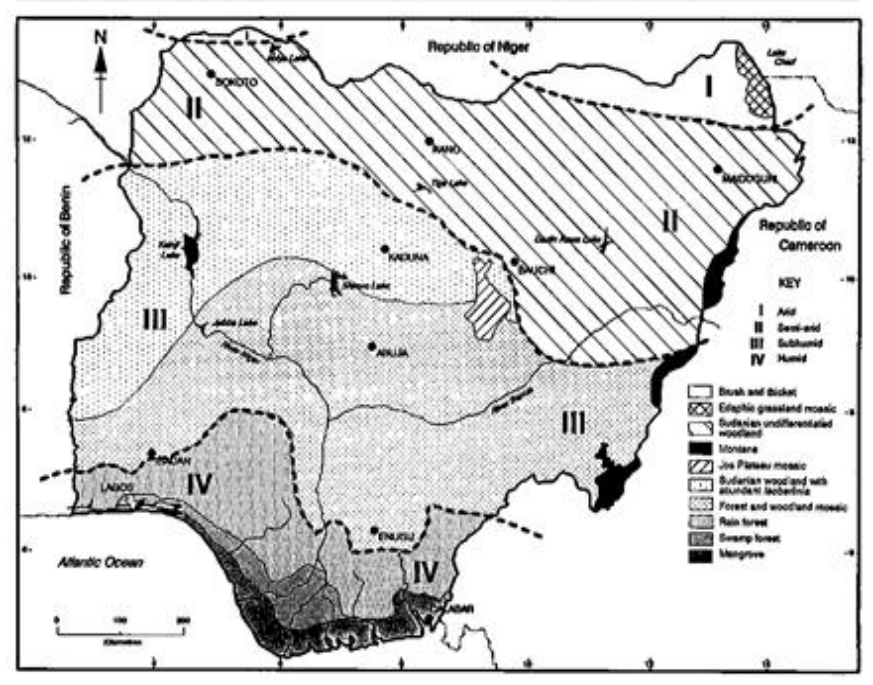

Figure 1 Map of Nigeria showing Vegetation and Climatic Zone of Study Area. (a) After Essien (2019) Pp. 76-77, (b) After White (1983) 


\subsection{Study design and sampling technique}

Twenty locations comprising fourty sampling points were randomly selected within the four Local Government Areas of Akoko division, Ondo State, Nigeria, as sampling sites. The sampling sites for the study was purposely selected to reflect (represent) as far as possible the Local Government Area of the study. In choosing the sites, consideration was also given to urbanization, accessibility and safety of the sampling (experimental materials) instruments among others [18].

At each site, a pollen trap (Modified Tauber Sampler) was mounted according to the methods of Tauber [19, 20], Pardoe et al. [21] and Giesecke et al. [22]. Prior to this, a mixture of glycerol (65 ml), formalin (30 ml) and phenol (5 ml) was poured into each of the trap. The positions of the traps at various locations were recorded using a Global Position System (GPS). The solutions in the trap prevented the palynomorphs from drying up, kill insects and also prevented the decay of dead organisms. The trap was left to stand throughout the duration of the study period. Fortnightly of each month, solution collection was done. The traps were washed with water to remove any contaminants and were then recharged with the above mentioned chemical solution. This procedure was repeated bi-monthly from October 2016 to September 2017 (dry season and rainy seasons' samples) for one year. The palynomorphs were recovered through centrifugation at 2000 r.p.m (revolution per minute) for 5 minutes and supernatant decanted each time. The precipitates were washed twice with distilled water and recovered through centrifugation. The sediments were treated with glacial acetic acid to remove water before acetolysis [23]. The recovered precipitates were washed with glacial acetic acid, and finally washed twice with distilled water, centrifuged each time and decanted. The recovered palynomorphs were stored in a plastic vials in glycerin and ethanol solution $(2: 1)$.

The palynomorphs were analysed palynologically and microscopically with Olympus microscope at x400 magnification for counting and Leica microscope at x1000 magnification for detailed morphological studies. Palynomorphs identification, counting and classification were done with the help of reference descriptions and photomicrographs from Agwu and Akanbi [25], Bonnefille and Riollet [26], Sowunmi [27] and Shubharani et al. [28]. In addition, prepared slides of pollen samples in the Palynological Research Unit; Department of Plant Science and Biotechnology, Adekunle Ajasin University, Akungba- Akoko, Nigeria were used.

\subsection{Statistical analysis of data}

The mean monthly pollen counts as well as the locations were subjected to analysis of variance (ANOVA). Means were separated using Duncan Multiple Range Test (DMRT) at $\mathrm{P}<0.05$ level of significance. All analyses were performed using the IBM SPSS Statistics Version 22.0.

\section{Results and discussion}

\subsection{Airborne pollen grains}

A total of 182 pollen types were identified across the study locations. These pollen types belong to 76 angiospermic plant families, 1gymnosperms and 4 pteridophytes. Out of the 76 plant families encountered, 68 families belong to the dicotyledonous plants, 6 to monocotyledonous plants, 1 gymnospermous plants and 1 was unidentified (Indeterminata) (Tables 1).

However, in absolute counts, the cumulative monthly pollen record showed that a total of 50,661 pollen grains were recorded with the highest monthly pollen counts of 9,297 recorded in November, followed by December $(8,416)$, October $(5,788)$, September $(5,600)$ and the least recorded in August $(1,926)$ and July $(1,530)$ respectively (Table 1). The predominant pollen types belong to the family Poaceae, Elaeis guineensis, Alchornea cordifolia, Aspilia africana, Casuarina equisetiifolia, and Tridax procumbens among others. 
Table 1 Cumulative monthly pollen grains counts for Akoko environment (October, 2016 - September, 2017)

\begin{tabular}{|c|c|c|c|c|c|c|c|c|c|c|c|c|c|c|}
\hline $\mathbf{S} / \mathbf{N}$ & Pollen type / Month & OCT & NOV & DEC & JAN. & FEB & MAR & APR & MAY & JUN & JUL & AUG & SEP & TOTAL \\
\hline A & DICOTYLEDONS & & & & & & & & & & & & & \\
\hline \multirow[t]{5}{*}{1} & ACANTHACEAE & & & & & & & & & & & & & \\
\hline & Anisotes sp. & 0 & 0 & 0 & 0 & 0 & 0 & 0 & 5 & 2 & 1 & 0 & 0 & 8 \\
\hline & Blepharis sp. & 3 & 0 & 0 & 3 & 0 & 0 & 0 & 0 & 0 & 0 & 0 & 5 & 11 \\
\hline & Hypoestes sp. & 0 & 8 & 0 & 0 & 0 & 0 & 0 & 0 & 0 & 0 & 0 & 0 & 8 \\
\hline & Justicia sp. & 1 & 5 & 0 & 0 & 0 & 4 & 1 & 3 & 4 & 1 & 2 & 0 & 21 \\
\hline 2 & AMARANTH./ CHENOPOD. & 26 & 28 & 24 & 16 & 11 & 158 & 35 & 53 & 26 & 9 & 3 & 26 & 415 \\
\hline \multirow[t]{6}{*}{3} & ANACARDIACEAE & & & & & & & & & & & & & \\
\hline & Anacardium occidentale & 0 & 6 & 4 & 2 & 0 & 0 & 0 & 12 & 0 & 0 & 0 & 0 & 24 \\
\hline & Antrocaryon micraster & 0 & 0 & 1 & 0 & 0 & 0 & 0 & 1 & 0 & 2 & 0 & 2 & 6 \\
\hline & Lannea acida & 0 & 121 & 231 & 0 & 0 & 91 & 181 & 71 & 0 & 33 & 51 & 195 & 974 \\
\hline & Mangifera indica & 0 & 0 & 0 & 0 & 0 & 0 & 9 & 3 & 0 & 0 & 0 & 0 & 12 \\
\hline & Spondias mombin & 1 & 2 & 0 & 2 & 0 & 1 & 2 & 0 & 2 & 0 & 0 & 0 & 10 \\
\hline \multirow[t]{2}{*}{4} & ANNONACEAE & & & & & & & & & & & & & \\
\hline & Annona senegalensis & 0 & 0 & 2 & 3 & 0 & 3 & 15 & 0 & 0 & 8 & 3 & 0 & 34 \\
\hline \multirow[t]{4}{*}{5} & APOCYNACEAE & & & & & & & & & & & & & \\
\hline & Alstonia boonei & 1 & 0 & 0 & 0 & 0 & 8 & 2 & 1 & 2 & 2 & 0 & 0 & 16 \\
\hline & Motandra sp. & 2 & 0 & 0 & 0 & 0 & 3 & 5 & 7 & 0 & 0 & 0 & 3 & 20 \\
\hline & Rauvolfia vomitoria & 0 & 0 & 0 & 0 & 14 & 9 & 0 & 0 & 0 & 0 & 0 & 0 & 23 \\
\hline \multirow[t]{4}{*}{6} & ASTERACEAE & & & & & & & & & & & & & \\
\hline & Aspilia africana & 415 & 446 & 31 & 66 & 18 & 10 & 4 & 17 & 20 & 39 & 104 & 77 & 1247 \\
\hline & Bidens pilosa & 3 & 21 & 11 & 4 & 2 & 0 & 0 & 0 & 0 & 0 & 3 & 5 & 49 \\
\hline & Emilia sonchifolia & 0 & 3 & 0 & 0 & 7 & 0 & 0 & 0 & 0 & 0 & 0 & 0 & 10 \\
\hline
\end{tabular}


Essien and Ige / GSC Biological and Pharmaceutical Sciences, 2020, 12(01), 116-137

Gutenbergia sp.

Tridax procumbens

Vernonia amygdalina

BIGNONIACEAE

Kigelia africana

Newbouldia laevis

Stereospermum kunthianum

Tecoma stans

8

\section{BOMBACACEAE}

Bombax buonopozense

Ceiba pentandra

9

\section{BORAGINACEAE}

Heliotropium sp.

10

\section{BURSERACEAE}

Canarium schweinfurthii Commiphora africana

11

\section{CAESALPINOIDEAE}

Afzelia africana

Berlinia grandifolia

Brachystergia eurycoma

Daniellia oliveri

Delonix regia

Detarium macrocarpum

Dialium guineense

Piliostigma thonningii

Senna occidentalis

\begin{tabular}{|c|c|c|c|c|c|c|c|c|c|c|c|c|}
\hline 3 & 0 & 0 & 0 & 0 & 0 & 28 & 54 & 552 & 4 & 1 & 0 & 642 \\
\hline 255 & 478 & 19 & 85 & 11 & 5 & 9 & 9 & 11 & 42 & 100 & 68 & 1092 \\
\hline 2 & 7 & 5 & 0 & 0 & 0 & 0 & 0 & 0 & 0 & 0 & 2 & 16 \\
\hline 3 & 0 & 14 & 0 & 0 & 0 & 0 & 0 & 0 & 0 & 0 & 8 & 25 \\
\hline 0 & 31 & 19 & 0 & 0 & 41 & 0 & 13 & 8 & 11 & 0 & 0 & 123 \\
\hline 0 & 7 & 3 & 14 & 0 & 0 & 0 & 0 & 0 & 0 & 0 & 0 & 24 \\
\hline 0 & 3 & 5 & 11 & 0 & 0 & 0 & 0 & 0 & 0 & 0 & 0 & 19 \\
\hline
\end{tabular}$$
25
$$

$0 \quad 0$

\begin{tabular}{|c|c|c|c|c|c|c|c|c|c|c|c|}
\hline 1 & 0 & 0 & 0 & 0 & 0 & 3 & 0 & 7 & 0 & 0 & 1 \\
\hline 0 & 12 & 10 & 0 & 0 & 7 & 19 & 38 & 0 & 0 & 0 & 0 \\
\hline
\end{tabular}

\begin{tabular}{|c|c|c|c|c|c|c|c|c|c|c|c|c|}
\hline 0 & 0 & 0 & 0 & 0 & 2 & 2 & 6 & 0 & 0 & 0 & 0 & 10 \\
\hline 0 & 8 & 5 & 21 & 0 & 4 & 18 & 0 & 0 & 0 & 0 & 0 & 56 \\
\hline 0 & 0 & 0 & 0 & 0 & 22 & 5 & 1 & 0 & 0 & 0 & 0 & 28 \\
\hline 34 & 11 & 19 & 0 & 5 & 9 & 1 & 8 & 0 & 0 & 25 & 14 & 126 \\
\hline 3 & 17 & 0 & 2 & 0 & 0 & 23 & 6 & 0 & 10 & 1 & 0 & 62 \\
\hline 0 & 0 & 0 & 0 & 0 & 0 & 0 & 0 & 3 & 12 & 7 & 0 & 22 \\
\hline 18 & 0 & 0 & 0 & 3 & 0 & 0 & 0 & 0 & 0 & 41 & 38 & 100 \\
\hline 12 & 8 & 0 & 0 & 0 & 0 & 0 & 0 & 0 & 2 & 4 & 0 & 26 \\
\hline 45 & 59 & 101 & 61 & 68 & 12 & 35 & 28 & 16 & 6 & 14 & 181 & 626 \\
\hline
\end{tabular}


Tessmannia sp.

CANNABACEAE (ULMACEAE)

Celtis sp.

CAPPARACEAE

Capparis sp.

Cleome sp.

14

CAPRIFOLIACEAE

Knautia sp.

Linnaea borealis

15

\section{CARICACEAE}

Carica papaya

CASUARINACEAE

Casuarina equisetiifolia

CELASTRACEAE

Maytenus sp.

\section{CHRYSOBALANACEAE}

Parinari curatellifolia

19

CLUSIACEAE

Garcinia kola

Symphonia globulifera

20

COCHLOSPERMACEAE

Cochlospermum planchonii

COMBRETACEAE

Combretum sp.

\section{COMMELINACEAE}

Aneilema sp.

\begin{tabular}{|c|c|c|c|c|c|c|c|c|c|c|c|c|}
\hline 0 & 5 & 3 & 0 & 0 & 3 & 7 & 0 & 0 & 0 & 0 & 11 & 29 \\
\hline 0 & 0 & 0 & 15 & 0 & 7 & 3 & 1 & 0 & 0 & 2 & 1 & 29 \\
\hline 0 & 0 & 0 & 0 & 0 & 0 & 5 & 1 & 0 & 0 & 0 & 0 & 6 \\
\hline 1 & 0 & 4 & 0 & 0 & 0 & 4 & 0 & 0 & 0 & 0 & 0 & 9 \\
\hline 3 & 0 & 0 & 0 & 0 & 0 & 0 & 0 & 0 & 5 & 2 & 0 & 10 \\
\hline 0 & 5 & 2 & 2 & 0 & 0 & 0 & 0 & 0 & 0 & 0 & 0 & 9 \\
\hline 0 & 0 & 0 & 0 & 0 & 0 & 0 & 0 & 0 & 0 & 11 & 31 & 42 \\
\hline 719 & 14 & 23 & 27 & 17 & 61 & 31 & 110 & 34 & 33 & 90 & 33 & 1192 \\
\hline 0 & 0 & 0 & 4 & 9 & 0 & 0 & 0 & 0 & 0 & 0 & 0 & 13 \\
\hline 0 & 0 & 0 & 0 & 0 & 31 & 5 & 11 & 9 & 0 & 0 & 0 & 56 \\
\hline 4 & 0 & 0 & 0 & 0 & 0 & 3 & 0 & 25 & 29 & 0 & 0 & 61 \\
\hline 0 & 2 & 5 & 0 & 0 & 8 & 12 & 5 & 0 & 0 & 0 & 2 & 34 \\
\hline 0 & 0 & 0 & 0 & 0 & 5 & 0 & 0 & 30 & 1 & 0 & 0 & 36 \\
\hline 11 & 42 & 30 & 24 & 47 & 40 & 31 & 42 & 42 & 12 & 20 & 35 & 376 \\
\hline 0 & 0 & 0 & 0 & 0 & 3 & 7 & 0 & 0 & 0 & 0 & 0 & 10 \\
\hline
\end{tabular}


CONNARACEAE

Cnestis ferruginea

CONVOLVULACEAE

Calycobolus sp.

Ipomoea sp.

Merremia sp.

CUCURBITACEAE

Cucumis sp.

Kedrostis africana

Luffa cylindrica

DICHAPETALACEAE

Dichapetalum sp.

27

DILLENIACEAE

Tetracera sp.

28

DIPTILOMIODEAE

Lepisanthes senegalensis

EBENACEAE

Diospyros mespiliformis

Diospyros sp.

30

ERICACEAE

Empetrum nigrum

Sapium sp.

31 EUPHORBIACEAE

Acalypha sp.

Alchornea cordifolia

Croton sp.

\begin{tabular}{|c|c|c|c|c|c|c|c|c|c|c|c|c|}
\hline 0 & 0 & 0 & 0 & 0 & 4 & 1 & 11 & 0 & 0 & 0 & 0 & 16 \\
\hline 0 & 0 & 0 & 0 & 4 & 1 & 0 & 0 & 0 & 0 & 0 & 0 & 5 \\
\hline 0 & 4 & 0 & 0 & 2 & 0 & 0 & 0 & 0 & 0 & 0 & 0 & 6 \\
\hline 0 & 2 & 0 & 0 & 0 & 6 & 0 & 0 & 12 & 0 & 0 & 4 & 24 \\
\hline 0 & 0 & 0 & 0 & 0 & 2 & 4 & 0 & 0 & 0 & 0 & 0 & 6 \\
\hline 0 & 0 & 0 & 0 & 0 & 2 & 6 & 0 & 0 & 0 & 0 & 0 & 8 \\
\hline 0 & 0 & 0 & 0 & 0 & 0 & 2 & 3 & 0 & 0 & 0 & 5 & 10 \\
\hline 0 & 0 & 0 & 0 & 0 & 0 & 0 & 2 & 3 & 0 & 0 & 0 & 5 \\
\hline 0 & 3 & 0 & 0 & 0 & 1 & 2 & 4 & 0 & 0 & 0 & 0 & 10 \\
\hline 0 & 0 & 0 & 0 & 0 & 6 & 9 & 0 & 0 & 0 & 0 & 0 & 15 \\
\hline 0 & 0 & 0 & 0 & 0 & 0 & 1 & 2 & 10 & 88 & 237 & 0 & 338 \\
\hline 0 & 0 & 0 & 0 & 0 & 0 & 2 & 4 & 8 & 0 & 0 & 0 & 14 \\
\hline 5 & 14 & 0 & 7 & 0 & 0 & 0 & 0 & 0 & 0 & 0 & 0 & 26 \\
\hline 0 & 0 & 7 & 0 & 0 & 0 & 0 & 0 & 0 & 0 & 0 & 3 & 10 \\
\hline 0 & 4 & 11 & 0 & 0 & 0 & 0 & 0 & 0 & 0 & 0 & 0 & 15 \\
\hline 15 & 847 & 497 & 196 & 99 & 202 & 99 & 56 & 29 & 7 & 14 & 65 & 2126 \\
\hline 0 & 0 & 2 & 4 & 0 & 9 & 3 & 0 & 0 & 0 & 0 & 0 & 18 \\
\hline
\end{tabular}


Euphorbia hirta

Euphorbia sp.

Jatropha curcas

Mallotus subulatus

Manihot esculenta

Ricinus communis

FAGACEAE

Fagus sp.

\section{GENTIANACEAE}

Gentianella amarella

\section{HYPERICACEAE} IRVINGIACEAE

Irvingia gabonensis

\section{LAMIACEAE}

Hyptis suaveolens

Leucas sp.

Ocimum sp.

Platostoma africanum

Vitex doniana

Vitex grandifolia

\section{LEEACEAE}

Leea guineensis

\section{LINACEAE}

Harungana madagascariensis

Solenostemon monostachyus

Linum catharticum

\begin{tabular}{|c|c|c|c|c|c|c|c|c|c|c|c|}
\hline 8 & 0 & 0 & 0 & 0 & 12 & 17 & 82 & 33 & 0 & 0 & 0 \\
\hline 0 & 0 & 1 & 0 & 1 & 3 & 3 & 0 & 1 & 0 & 0 & 0 \\
\hline 2 & 0 & 0 & 0 & 0 & 0 & 8 & 0 & 0 & 2 & 2 & 0 \\
\hline 0 & 0 & 0 & 0 & 4 & 2 & 0 & 0 & 0 & 0 & 0 & 0 \\
\hline 0 & 0 & 0 & 0 & 0 & 1 & 2 & 4 & 1 & 0 & 0 & 0 \\
\hline 0 & 0 & 0 & 0 & 0 & 0 & 8 & 6 & 3 & 0 & 0 & 0 \\
\hline
\end{tabular}

4

4

\section{3}

15

$202 \quad 29$

$21 \quad 20$

$47 \quad 7$

7

(1)

$\begin{array}{lllllllllll}76 & 24 & 6 & 27 & 9 & 10 & 4 & 1 & 0 & 205 & 421 \\ 9 & 2 & 1 & 0 & 0 & 0 & 2 & 9 & 16 & 7 & 58 \\ 0 & 0 & 0 & 0 & 0 & 0 & 3 & 5 & 18 & 31 & 128 \\ 0 & 0 & 0 & 0 & 0 & 0 & 0 & 0 & 0 & 3 & 11 \\ 0 & 0 & 6 & 0 & 0 & 0 & 0 & 0 & 0 & 0 & 9 \\ 6 & 2 & 18 & 0 & 0 & 0 & 0 & 0 & 0 & 21 & 63 \\ 0 & 0 & 0 & 0 & 0 & 2 & 26 & 44 & 0 & 0 & 72\end{array}$


Linum sp.

39

LOGANIACEAE

Anthocleista djalonensis

Spigelia anthelmia

40

MALVACEAE

Abelmoschus esculentus

Corchorus olitorius

Nesogordonia papaverifera

Sida acuta

41

\section{MELASTOMATACEAE}

Dissotis sp.

42

\section{MELIACEAE}

Azadirachta indica

Khaya ivorensis

Khaya senegalensis

Trichilia prieureana

43

MIMOSOIDEAE

Acacia senegal

Acacia sp.

Adenanthera pavonina

Albizia sp.

Albizia zygia

Aubrevillea kerstingii

Leucaena leucocephala

Mimosa pigra

Parkia biglobosa

\begin{tabular}{|c|c|c|c|c|c|c|c|c|c|c|c|c|}
\hline 12 & 0 & 0 & 1 & 3 & 1 & 1 & 9 & 2 & 5 & 8 & 0 & 42 \\
\hline 0 & 0 & 13 & 3 & 3 & 2 & 2 & 5 & 2 & 1 & 0 & 0 & 31 \\
\hline 5 & 0 & 5 & 0 & 0 & 0 & 0 & 0 & 0 & 0 & 0 & 2 & 12 \\
\hline 0 & 0 & 21 & 0 & 0 & 0 & 0 & 0 & 0 & 0 & 0 & 9 & 30 \\
\hline 0 & 3 & 0 & 0 & 2 & 6 & 0 & 0 & 0 & 0 & 0 & 0 & 11 \\
\hline 11 & 0 & 21 & 0 & 0 & 0 & 0 & 0 & 0 & 0 & 0 & 9 & 41 \\
\hline 24 & 308 & 18 & 49 & 162 & 2 & 9 & 3 & 19 & 1 & 34 & 12 & 641 \\
\hline 41 & 0 & 0 & 0 & 0 & 0 & 0 & 0 & 11 & 26 & 21 & 13 & 112 \\
\hline 0 & 2 & 9 & 0 & 0 & 5 & 6 & 1 & 7 & 5 & 6 & 2 & 43 \\
\hline 0 & 2 & 7 & 3 & 0 & 0 & 0 & 0 & 0 & 0 & 0 & 4 & 16 \\
\hline 4 & 15 & 2 & 7 & 7 & 23 & 63 & 9 & 1 & 8 & 9 & 1 & 149 \\
\hline 0 & 3 & 3 & 0 & 0 & 3 & 7 & 0 & 0 & 0 & 2 & 0 & 18 \\
\hline 0 & 3 & 8 & 0 & 0 & 7 & 14 & 5 & 0 & 0 & 0 & 0 & 37 \\
\hline 0 & 0 & 0 & 0 & 0 & 0 & 0 & 0 & 0 & 3 & 0 & 0 & 3 \\
\hline 0 & 0 & 0 & 0 & 0 & 2 & 6 & 2 & 1 & 0 & 0 & 0 & 11 \\
\hline 54 & 0 & 0 & 0 & 0 & 0 & 5 & 24 & 2 & 6 & 25 & 0 & 116 \\
\hline 0 & 191 & 151 & 177 & 33 & 0 & 0 & 0 & 13 & 17 & 0 & 0 & 582 \\
\hline 7 & 11 & 6 & 8 & 0 & 0 & 0 & 0 & 0 & 0 & 0 & 16 & 48 \\
\hline 0 & 21 & 5 & 0 & 0 & 0 & 0 & 0 & 0 & 0 & 0 & 3 & 29 \\
\hline 0 & 0 & 0 & 19 & 11 & 0 & 121 & 53 & 28 & 0 & 0 & 0 & 232 \\
\hline 15 & 137 & 225 & 39 & 18 & 0 & 11 & 182 & 14 & 5 & 4 & 78 & 728 \\
\hline
\end{tabular}


Essien and Ige / GSC Biological and Pharmaceutical Sciences, 2020, 12(01), 116-137

Pentaclethra macrophylla

Prosopis africana

44

MORACEAE

Ficus exasperata

Milicia excelsa

Morus sp.

Treculia africana

45

MYRISTICACEAE

Pycnanthus angolensis

MYRTACEAE

Euginea sp.

Psidium guajava

Syzygium guineense

47

OCHNACEAE

Lophira alata

OLACACEAE

Olax subcorpioidea

Olax sp.

49

PANDACEAE

Microdesmis sp.

50

PAPILIONOIDEAE
Eucalyptus globulus

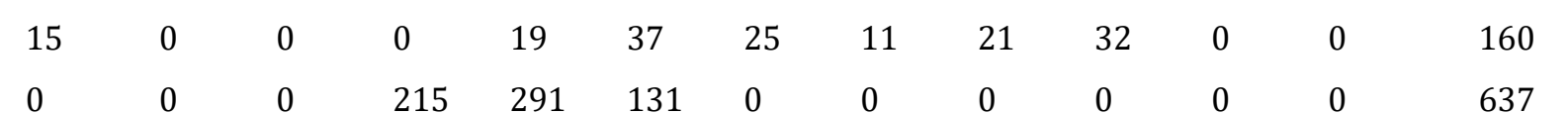

$\begin{array}{lllllllllllll}0 & 49 & 141 & 160 & 0 & 253 & 6 & 12 & 5 & 0 & 0 & 19 & 645\end{array}$

$\begin{array}{llllllllllll}0 & 0 & 0 & 0 & 0 & 10 & 4 & 0 & 0 & 0 & 0 & 0\end{array}$

$\begin{array}{llllllllllll}0 & 0 & 0 & 0 & 0 & 5 & 0 & 0 & 0 & 0 & 0 & 0\end{array}$

$\begin{array}{llllllllllll}0 & 61 & 21 & 32 & 0 & 0 & 0 & 0 & 0 & 0 & 0 & 10\end{array}$

$\begin{array}{lllllllllllll}16 & 0 & 0 & 0 & 0 & 0 & 0 & 0 & 0 & 3 & 7 & 0 & 26\end{array}$

$\begin{array}{lllllllllllll}57 & 61 & 0 & 1 & 3 & 1 & 27 & 94 & 13 & 10 & 41 & 16 & 324\end{array}$

$\begin{array}{rlllllllllll}0 & 0 & 0 & 0 & 2 & 0 & 0 & 0 & 3 & 0 & 0 & 0\end{array}$

$\begin{array}{lllllllllllll}7 & 11 & 3 & 8 & 0 & 48 & 22 & 159 & 12 & 4 & 12 & 14 & 300\end{array}$

$\begin{array}{lllllllllllll}4 & 9 & 41 & 6 & 10 & 15 & 14 & 14 & 2 & 0 & 4 & 4 & 123\end{array}$

$\begin{array}{lllllllllllll}2 & 0 & 0 & 1 & 3 & 0 & 0 & 0 & 0 & 0 & 0 & 0 & 6\end{array}$

$\begin{array}{lllllllllllll}0 & 0 & 5 & 6 & 4 & 2 & 0 & 0 & 0 & 0 & 2 & 4 & 23\end{array}$

Baphia sp.

Dalbergia sp.

Indigofera sp.

Pterocarpus osum

(3)

5

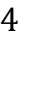
in

\section{3}

3

$\begin{array}{lllllllllllll}1 & 5 & 13 & 11 & 0 & 36 & 21 & 10 & 0 & 0 & 1 & 2 & 100 \\ 0 & 0 & 0 & 0 & 0 & 11 & 9 & 2 & 0 & 0 & 0 & 1 & 23 \\ 0 & 4 & 5 & 0 & 0 & 0 & 0 & 0 & 0 & 0 & 0 & 0 & 9 \\ 0 & 8 & 0 & 0 & 0 & 12 & 3 & 7 & 11 & 0 & 0 & 0 & 41 \\ 0 & 3 & 2 & 0 & 3 & 1 & 3 & 0 & 2 & 0 & 0 & 0 & 14\end{array}$


Rhynchosia sp.

Vigna unguiculata

Periploca sp.

PHYLLANTHACEAE

Antidesma sp.

Bridelia ferruginea

Hymenocardia acida

Phyllanthus sp.

Securinega virosa

Uapaca sp.

PLUMBAGINACEAE

Plumbago zeylanica

56

POLYGALACEAE

Polygala sp.

57

PORTULACACEAE

Talinum triangulare

58

PROTEACEAE

Protea elliotii

Protea madiensis

59

\begin{tabular}{|c|c|c|c|c|c|c|c|c|c|c|c|c|}
\hline 0 & 0 & 0 & 0 & 0 & 5 & 4 & 3 & 0 & 0 & 0 & 0 & 12 \\
\hline 11 & 0 & 9 & 0 & 0 & 0 & 0 & 0 & 0 & 0 & 0 & 5 & 25 \\
\hline 0 & 0 & 0 & 0 & 0 & 0 & 1 & 4 & 2 & 0 & 0 & 0 & 7 \\
\hline 0 & 72 & 15 & 15 & 0 & 0 & 0 & 0 & 0 & 0 & 0 & 125 & 227 \\
\hline 0 & 283 & 0 & 224 & 12 & 0 & 1 & 0 & 0 & 0 & 0 & 4 & 524 \\
\hline 27 & 3 & 401 & 4 & 2 & 8 & 2 & 2 & 7 & 4 & 0 & 14 & 474 \\
\hline 0 & 0 & 5 & 0 & 0 & 2 & 2 & 2 & 0 & 0 & 0 & 2 & 13 \\
\hline 2 & 30 & 8 & 78 & 18 & 20 & 40 & 229 & 8 & 7 & 7 & 6 & 453 \\
\hline 4 & 0 & 9 & 24 & 10 & 25 & 15 & 11 & 13 & 10 & 8 & 6 & 135 \\
\hline 10 & 27 & 12 & 16 & 11 & 5 & 2 & 3 & 5 & 5 & 8 & 8 & 112 \\
\hline 1 & 1 & 0 & 0 & 0 & 0 & 8 & 0 & 26 & 2 & 5 & 0 & 43 \\
\hline 11 & 0 & 0 & 0 & 0 & 0 & 0 & 0 & 0 & 0 & 3 & 7 & 21 \\
\hline 5 & 21 & 15 & 18 & 0 & 0 & 0 & 0 & 0 & 0 & 8 & 10 & 77 \\
\hline 18 & 12 & 8 & 0 & 0 & 0 & 0 & 0 & 0 & 0 & 5 & 5 & 48 \\
\hline 1 & 1 & 0 & 3 & 0 & 0 & 0 & 0 & 0 & 0 & 1 & 0 & 6 \\
\hline 0 & 0 & 0 & 0 & 3 & 0 & 0 & 0 & 0 & 0 & 0 & 0 & 3 \\
\hline
\end{tabular}

Lasiodiscus sp. 
ROSACEAE

Fillipendula ulmaria

RUBIACEAE

Borreria sp.

Canthium sp.

Crossopteryx febrifuga

Gaertnera paniculata

Gardenia sp.

Morelia senegalensis

Nauclea latifolia

62

\section{RUTACEAE}

Citrus sp.

Zanthoxylum zanthoxyloides

63

SAPINDACEAE

Allophyllus sp.

Blighia unijugata

Paullinia pinnata

64

SAPOTACEAE

Vitellaria paradoxa

SOLANACEAE

Physalis angulata

Solanum melongena

Solanum torvum

66

STERCULIACEAE

Cola sp.

Sterculia sp.

\begin{tabular}{|c|c|c|c|c|c|c|c|c|c|c|c|c|}
\hline 7 & 11 & 3 & 8 & 0 & 48 & 22 & 159 & 12 & 4 & 12 & 14 & 300 \\
\hline 0 & 0 & 0 & 0 & 0 & 10 & 4 & 0 & 0 & 0 & 0 & 0 & 14 \\
\hline 16 & 0 & 0 & 0 & 0 & 5 & 0 & 0 & 0 & 3 & 7 & 0 & 31 \\
\hline 0 & 34 & 5 & 13 & 0 & 0 & 0 & 0 & 0 & 0 & 0 & 3 & 55 \\
\hline 0 & 22 & 9 & 10 & 0 & 0 & 0 & 0 & 0 & 0 & 0 & 5 & 46 \\
\hline 0 & 5 & 7 & 9 & 0 & 0 & 0 & 0 & 0 & 0 & 0 & 2 & 23 \\
\hline 0 & 49 & 141 & 160 & 0 & 253 & 6 & 12 & 5 & 0 & 0 & 19 & 645 \\
\hline 57 & 1 & 0 & 1 & 5 & 27 & 61 & 94 & 16 & 10 & 41 & 16 & 329 \\
\hline 4 & 7 & 35 & 6 & 10 & 12 & 14 & 9 & 2 & 0 & 4 & 4 & 107 \\
\hline 0 & 2 & 6 & 0 & 0 & 3 & 0 & 5 & 0 & 0 & 0 & 0 & 16 \\
\hline 0 & 0 & 5 & 6 & 4 & 2 & 0 & 0 & 0 & 0 & 2 & 4 & 23 \\
\hline 0 & 0 & 0 & 0 & 0 & 0 & 5 & 11 & 3 & 0 & 0 & 0 & 19 \\
\hline 2 & 0 & 0 & 1 & 3 & 0 & 11 & 36 & 21 & 0 & 0 & 0 & 74 \\
\hline 1 & 5 & 11 & 7 & 0 & 27 & 18 & 10 & 0 & 0 & 1 & 2 & 82 \\
\hline 1 & 1 & 0 & 0 & 0 & 0 & 8 & 0 & 26 & 2 & 5 & 0 & 43 \\
\hline 27 & 3 & 401 & 4 & 2 & 8 & 2 & 2 & 7 & 4 & 0 & 14 & 474 \\
\hline 0 & 0 & 5 & 0 & 0 & 2 & 2 & 2 & 0 & 0 & 0 & 2 & 13 \\
\hline 4 & 0 & 9 & 24 & 10 & 25 & 15 & 11 & 13 & 10 & 8 & 6 & 135 \\
\hline 10 & 27 & 12 & 16 & 11 & 5 & 2 & 3 & 5 & 5 & 8 & 8 & 112 \\
\hline
\end{tabular}




\begin{tabular}{|c|c|c|c|c|c|c|c|c|c|c|c|c|c|c|}
\hline & Triplochiton scleroxylon & 2 & 102 & 23 & 93 & 18 & 20 & 40 & 229 & 8 & 7 & 7 & 131 & 680 \\
\hline \multirow[t]{4}{*}{67} & URTICACEAE & & & & & & & & & & & & & \\
\hline & Musanga cecropioides & 3 & 21 & 10 & 8 & 0 & 0 & 0 & 0 & 0 & 0 & 8 & 7 & 57 \\
\hline & Tieghemella africana & 11 & 0 & 0 & 0 & 0 & 0 & 0 & 0 & 0 & 0 & 3 & 7 & 21 \\
\hline & Trema guineense & 12 & 4 & 10 & 10 & 0 & 0 & 0 & 0 & 0 & 0 & 3 & 5 & 44 \\
\hline \multirow[t]{6}{*}{68} & VERBENACEAE & & & & & & & & & & & & & \\
\hline & Gmelina arborea & 8 & 8 & 3 & 0 & 0 & 0 & 0 & 0 & 0 & 0 & 2 & 3 & 24 \\
\hline & Stachytarpheta sp. & 1 & 1 & 0 & 3 & 0 & 0 & 0 & 0 & 0 & 0 & 1 & 0 & 6 \\
\hline & Tectona grandis & 0 & 0 & 0 & 0 & 3 & 0 & 0 & 0 & 0 & 0 & 0 & 0 & 3 \\
\hline & SUB TOTAL & 2300 & 3970 & 3099 & 2248 & 1078 & 2184 & 1433 & 2206 & 1330 & 667 & 1141 & 1769 & 23425 \\
\hline & 2.MONOCOTYLEDONS: & & & & & & & & & & & & & \\
\hline \multirow[t]{4}{*}{69} & ARECACEAE & & & & & & & & & & & & & \\
\hline & Cocos nucifera & 0 & 0 & 5 & 2 & 5 & 15 & 5 & 10 & 2 & 0 & 0 & 0 & 44 \\
\hline & Elaeis guineensis & 350 & 1411 & 1148 & 395 & 247 & 622 & 594 & 320 & 146 & 123 & 187 & 379 & 5922 \\
\hline & Raphia sp. & 0 & 0 & 0 & 3 & 6 & 2 & 1 & 0 & 0 & 0 & 0 & 0 & 12 \\
\hline \multirow[t]{2}{*}{70} & COLCHICACEAE & & & & & & & & & & & & & \\
\hline & Gloriosa superba & 0 & 0 & 0 & 0 & 0 & 5 & 7 & 4 & 2 & 0 & 3 & 0 & 21 \\
\hline \multirow[t]{2}{*}{71} & COSTACEAE & & & & & & & & & & & & & \\
\hline & Costus afer & 0 & 2 & 0 & 1 & 0 & 0 & 0 & 3 & 2 & 5 & 0 & 0 & 13 \\
\hline 72 & CYPERACEAE & 19 & 13 & 12 & 10 & 9 & 17 & 10 & 116 & 42 & 25 & 22 & 26 & 321 \\
\hline \multirow[t]{2}{*}{73} & DIOSCOREACEAE & & & & & & & & & & & & & \\
\hline & Dioscorea sp. & 0 & 0 & 0 & 0 & 0 & 8 & 3 & 1 & 0 & 0 & 0 & 2 & 14 \\
\hline 74 & POACEAE & 3104 & 3892 & 4145 & 1679 & 594 & 551 & 567 & 889 & 699 & 703 & 565 & 3423 & 20811 \\
\hline \multirow[t]{2}{*}{75} & INDETERMINATA & 1 & 2 & 7 & 1 & 6 & 1 & 4 & 4 & 1 & 5 & 3 & 4 & 39 \\
\hline & SUB TOTAL & 3479 & 5320 & 5317 & 2091 & 867 & 1221 & 1190 & 1347 & 894 & 861 & 780 & 3829 & 27197 \\
\hline B & GYMNOSPERMS & & & & & & & & & & & & & \\
\hline
\end{tabular}


PINACEAE

\begin{tabular}{llllllllllllll} 
Pinus caribaea & 9 & 7 & 0 & 4 & 0 & 1 & 4 & 4 & 1 & 2 & 5 & 2 & 39 \\
\hline TOTAL POLLEN COUNTS & 5788 & 9297 & 8416 & 4343 & 1945 & 3406 & 2628 & 3557 & 2225 & 1530 & 1926 & 5600 & 50661
\end{tabular}

C PTERIDOPHYTES:

1. ASPIDIACEAE

Cystopteris fragilis

Goniopteris vivipara

\begin{tabular}{|c|c|c|c|c|c|c|c|c|c|c|c|}
\hline 0 & 0 & 0 & 0 & 0 & 0 & 0 & 0 & 0 & 2 & 4 & 0 \\
\hline 0 & 0 & 0 & 0 & 0 & 0 & 22 & 0 & 0 & 0 & 0 & 0 \\
\hline
\end{tabular}

2. DAVALLIACEAE

Nephrolepis exaltata

3. HYMENOPHYLLACEAE

Callistopteris apiifolia

4. PTERIDACEAE

Anopteris hexagona

Pteris dentata

Monolete

Trilete

0

0

0

$\begin{array}{llllllllllll}0 & 0 & 0 & 0 & 0 & 0 & 2 & 3 & 0 & 0 & 0 & 0\end{array}$

SUB TOTAL

$\begin{array}{llllllllllll}0 & 0 & 0 & 0 & 0 & 0 & 0 & 3 & 1 & 0 & 0 & 0\end{array}$

$\begin{array}{lllllllllllll}0 & 0 & 0 & 0 & 0 & 0 & 2 & 2 & 0 & 0 & 0 & 0 & 4\end{array}$

$\begin{array}{llllllllllll}126 & 113 & 23 & 22 & 18 & 8 & 36 & 39 & 36 & 23 & 22 & 411\end{array}$

GRAND TOTAL

$\begin{array}{llllllllllll}1 & 1 & 2 & 0 & 1 & 14 & 2 & 5 & 17 & 0 & 0 & 11\end{array}$

GRAND TOTAL

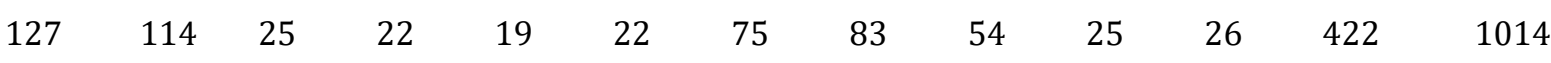

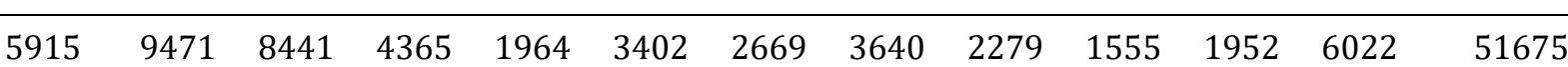


Statistical analysis of the mean monthly pollen counts showed that there was a significant difference $(\mathrm{P}<0.05)$ in the monthly pollen abundance. Multiple comparison using DMRT showed that the quantity of pollen counts recorded in November was significantly different $(\mathrm{P}<0.05)$ from those recorded in the months of March, April, May, June, July, August, September, October, January and February, but not significantly different from the pollen counts recorded in December (Table 2).

Table 2 Mean monthly pollen counts recorded in the study

\begin{tabular}{lll}
\hline $\mathbf{S} / \mathbf{N}$ & Months & Mean pollen grains \pm S.E \\
\hline 1 & October & $1.55 \pm 0.21^{\mathrm{d}}$ \\
2 & November & $2.46 \pm 0.27^{\mathrm{e}}$ \\
3 & December & $2.23 \pm 0.28^{\mathrm{e}}$ \\
4 & January & $1.18 \pm 0.12^{\mathrm{cd}}$ \\
5 & February & $0.52 \pm 0.05^{\mathrm{ab}}$ \\
6 & March & $0.89 \pm 0.08^{\mathrm{abc}}$ \\
7 & April & $0.70 \pm 0.07^{\mathrm{abc}}$ \\
8 & May & $0.96 \pm 0.14^{\mathrm{bc}}$ \\
9 & June & $0.59 \pm 0.12^{\mathrm{ab}}$ \\
10 & July & $0.42 \pm 0.09^{\mathrm{a}}$ \\
11 & August & $0.51 \pm 0.07^{\mathrm{ab}}$ \\
12 & September & $1.48 \pm 0.24^{\mathrm{d}}$ \\
& pValue & $0.000^{*}$ \\
\hline
\end{tabular}

The study also showed that the airborne pollen grains were unevenly distributed throughout the year with many sporadically dispersed monthly and at the study locations (Fig. 2 and 3).

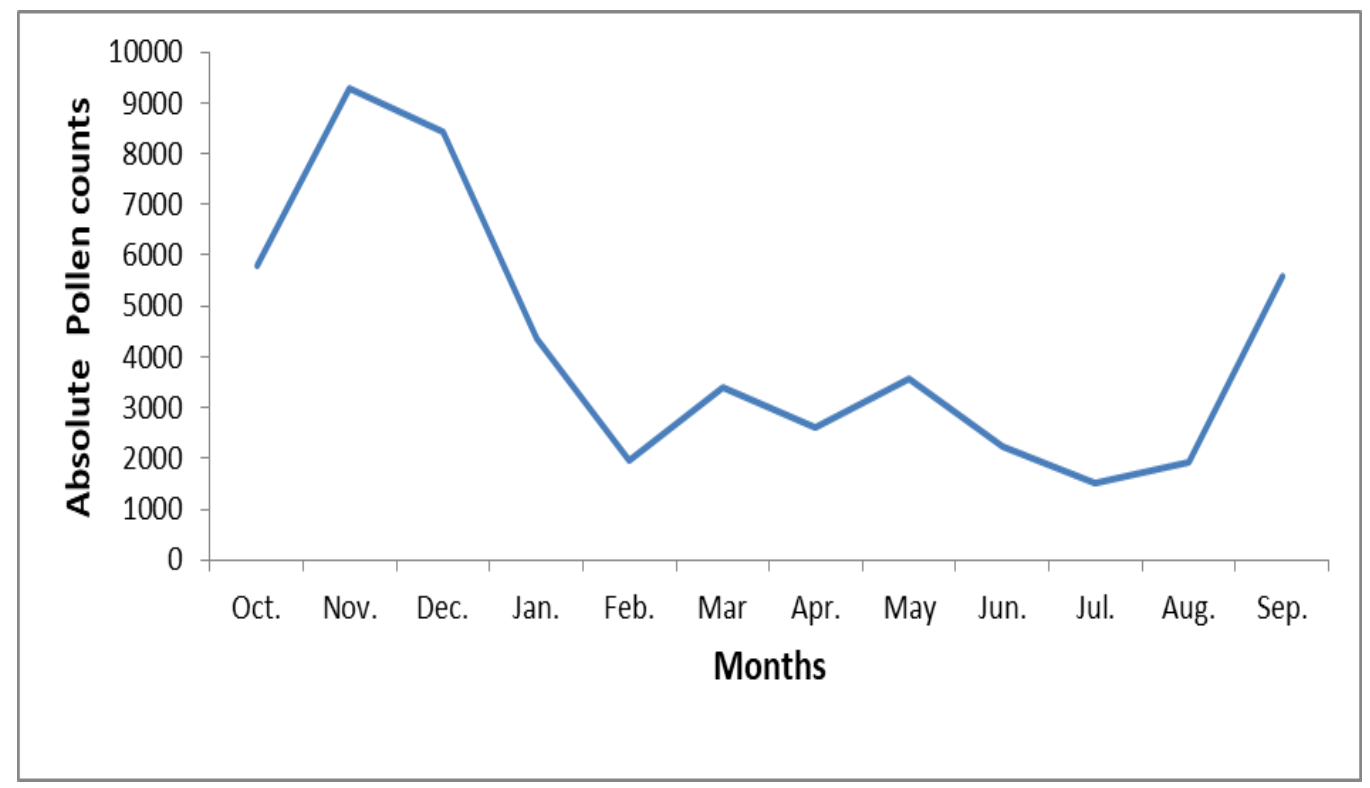

Figure 2 Variation in cumulative monthly pollen counts recorded across the study period 
The analysis of variance also showed that there was significant difference in the mean pollen counts recorded across the study locations. Multiple comparisons using Duncan Multiple Range Test (DMRT) showed that the highest mean pollen count was recorded in Ayegunle $\left(2.07 \pm 0.45^{\mathrm{d}}\right)$ and was significantly different from those recorded for all other study locations except Ipesi, Ifira and Ipe (Table 3).

Table 3 Mean pollen grains counts recorded at the study locations

\begin{tabular}{|c|c|c|}
\hline $\mathbf{S} / \mathbf{N}$ & Study location & Mean pollen grains $\pm S . E$ \\
\hline 1 & Ayegunle & $2.07 \pm 0.45^{\mathrm{d}}$ \\
\hline 2 & Ipesi & $1.64 \pm 0.25^{\mathrm{cd}}$ \\
\hline 3 & Ipe & $1.54 \pm 0.27 \mathrm{bcd}$ \\
\hline 4 & Ifira & $1.51 \pm 0.21^{\mathrm{abcd}}$ \\
\hline 5 & Akungba & $1.31 \pm 0.25^{\mathrm{abc}}$ \\
\hline 6 & Isua & $1.28 \pm 0.20^{\mathrm{abc}}$ \\
\hline 7 & Akunnu & $1.19 \pm 0.18^{\mathrm{abc}}$ \\
\hline 8 & Iboropa & $1.14 \pm 0.19 \mathrm{abc}$ \\
\hline 9 & Auga & $1.05 \pm 0.17^{\mathrm{abc}}$ \\
\hline 10 & Oba & $0.97 \pm 0.19 \mathrm{abc}$ \\
\hline 11 & Irun & $0.97 \pm 0.19 \mathrm{abc}$ \\
\hline 12 & Sosan & $0.97 \pm 0.17^{\mathrm{abc}}$ \\
\hline 13 & Ikare & $0.94 \pm 0.18 \mathrm{abc}$ \\
\hline 14 & Ikaram & $0.94 \pm 0.17^{\mathrm{abc}}$ \\
\hline 15 & Oke-Oka & $0.89 \pm 0.18^{a b}$ \\
\hline 16 & Ise & $0.86 \pm 0.17^{\mathrm{ab}}$ \\
\hline 17 & Supare & $0.86 \pm 0.16^{\mathrm{ab}}$ \\
\hline 18 & Arigidi & $0.80 \pm 0.15^{\mathrm{a}}$ \\
\hline 19 & Ogbagi & $0.79 \pm 0.18 \mathrm{a}$ \\
\hline \multirow[t]{2}{*}{20} & Oke-Agbe & $0.78 \pm 0.15^{\mathrm{a}}$ \\
\hline & p Value & $0.000 *$ \\
\hline
\end{tabular}

Among the classified plant families, the maximum mean number of pollen counts of $86.71 \pm 5.74$ was recorded for Poaceae followed by $8.29 \pm 0.66$ in Arecaceae, $4.97 \pm 0.99$ in Casuarinaceae and $2.12 \pm 0.29$ in Asteraceae and the least with $0.02 \pm 0.01$ in Proteaceae, $0.02 \pm 0.01$ in Hymenophyllaceae as well as $0.01 \pm 0.01$ in Boraginaceae and $0.01 \pm 0.01$ in Pteridaceae. Of the individual plant species Elaeis guineensis contributed the maximum mean number of pollen counts (24.65 \pm 1.52$)$ followed by Alchornea cordifolia (9.25 \pm 1.21$)$, Aspilia africana (5.19 \pm 1.06$)$, Casuarina equisetiifolia (4.97 \pm 0.99$)$, Tridax procumbens $(4.55 \pm 1.26)$, Lannea acida $(4.06 \pm 0.55)$ and the least by Heliotropium $\mathrm{sp}$. (0.01 \pm 0.01$)$, Capparis sp. (0.01 \pm 0.01$)$, Protea madiensis $(0.01 \pm 0.01)$, Tectona grandis $(0.01 \pm 0.01)$ and Anopteris hexagona $(0.00 \pm 0.00)$.

There were noticeable monthly increase and decrease in the quantity of pollen grains and other palynomorphs counted. The pollen load of the entire study area varied quantitatively and qualitatively not only from month-to-month but also from location-to-location. Variations in cumulative pollen counts recorded across the study locations are shown in Figure 3. 


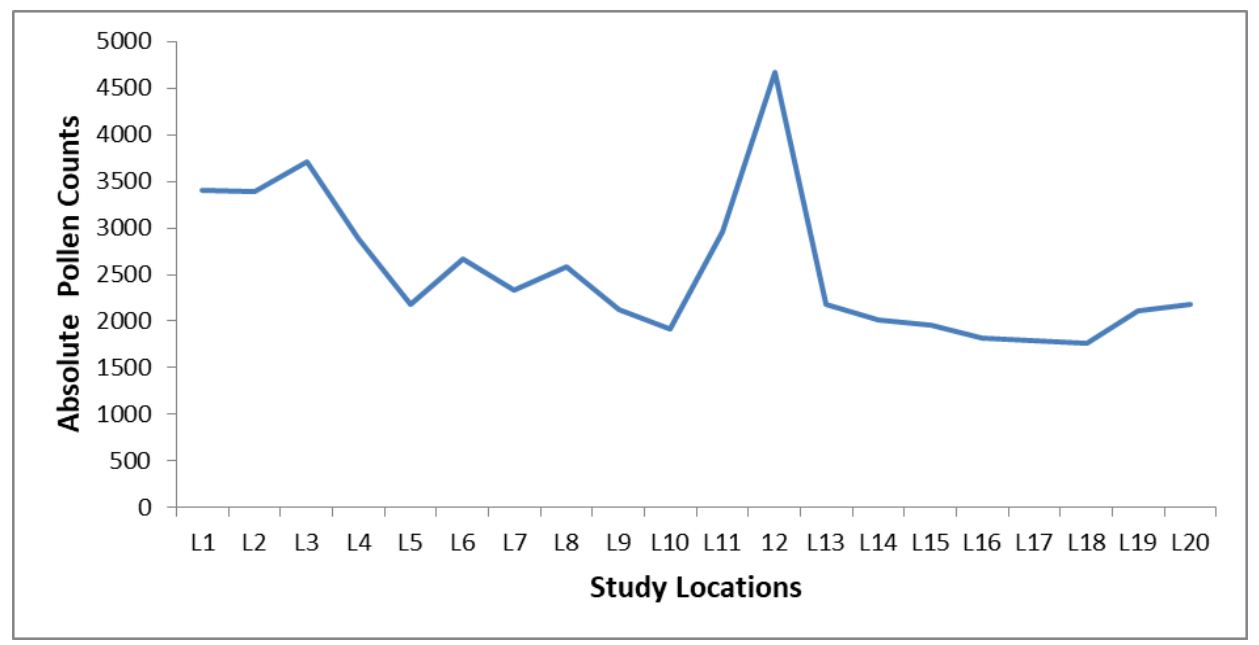

Figure 3 Variation in cumulative pollen counts recorded across the study locations

L1=Ifira, L2=Ipe, L3=Ipesi, L4=Isua, L5=Sosan, L6=Akunnu, L7=Auga, L8=Iboropa, L9=Ikare, 10=Ise, L11=Akungba, L12=Ayegunle, L13=Oba, L14=0ke-Oka, L15=Supare, L16=Arigidi, L17=0gbagi, L18=0ke-Agbe, L19=Ikaram, L20=Irun.

With reference to the monthly pollen abundance in the atmosphere, the results showed that the concentration of pollen grains in the atmosphere fluctuates considerably with the seasons. The monthly airborne pollen concentrations showed three discernible periods of pollen variability in the atmosphere. The three periods of pollen abundance in the atmosphere showed that the highest mean concentration occurred during the late rainy season to early dry season followed by the period of dry season to early rainy season and the least occurred during the mid-rainy season (Table 4).

Table 4 Season of pollen abundance in the atmosphere

\begin{tabular}{llll}
\hline S/N & Seasons & Months & Pollen counts \\
\hline 1 & Late rainy season to early dry season & September to January & 33,444 \\
2 & Dry season to early rainy season & February to June & 13,761 \\
3 & Mid rainy season & July to August & 3,456 \\
& Cumulative total pollen counts & & 50,661 \\
\hline
\end{tabular}

\subsection{Distribution and diversity of airborne pollen grains}

Airborne pollen grain exhibits seasonal fluctuations in quantity as well as composition. Unspecified numbers and type of pollen grains remain airborne after the flowering season. It is also possible that pollen grains that had already fallen on the ground were refloated or these extra seasonal pollen grains may have come from plants which continued flowering out of the season, examples are Poaceae and Elaeis guineensis.

Elaeis guineensis which is a major pollen contributor in this study is found in all forms of sub-types vegetation in Akoko environment, Ondo State ranging from urban flora to grasslands, farmlands, woodlands and mature nature forests. Akoko people are known for the cultivation and production of oil palm and palm produce. Because of their enormous economic benefits, they are protected and established in farms and plantations. They flower virtually all year round, hence the characteristic increase and decrease in pollen content; turning out large quantities of pollen grains particularly at the major flowering periods. Its presence in the pollen spectra of the study area depicts extension of wooded grassland and traditional forest.

The aerodynamic properties of the pollen grains promote their dispersal and distribution in the turbulent air mass. This accounts for part of the reason why the pollen grains of Elaeis guineensis were trapped in the atmosphere throughout the twelve (12) months of study and at each sampling site at least in reasonable proportion. Findings corroborated favourably by the report of Njokuocha [29].

The grass family is reported to be the major contributor to airborne pollen assemblage in most aeropalynological studies [12] [16] [30] [31]. Mabberley [32] in Latorre and Belmonte [33] reported that the Poaceae family has a 
cosmopolitan distribution representing $20 \%$ of the world's vegetation cover. With the light microscope, it is difficult to separate the Poaceae pollen on the species level.

However, the increase of grass pollen could also be attributed to the fact that most members of this family flower and shed their floral parts and the subsequent shriveling of these flowers eventually makes the entire plant to dry up as the environment approaches extreme dryness and destruction by annual bush burning thereby liberating their pollen into the atmosphere.

Poaceae pollen is present in the atmosphere nearly all year round, but the highest concentrations were observed between the months of September and November- February. The highest concentration of Poaceae pollen could also be attributed to high density and luxuriant growth of some of these grasses such as Panicum maximum, Andropogon tectorum, Loudetia arundinacea, Schizachyrium brevifolium, Hyparrhenia barteri, Pennisetum purpureum, Imperata cylindica and seasonal cultivated species such as Zea mays, among others, which flower more or less throughout the year and produce enormous amount of pollen grains [34 - 38]. The anemophilous mode of pollination, coupled with good aerodynamic properties of the pollen grains are some of the inherent factors that account for the higher Poaceae pollen concentration.

Alchornea cordifolia is associated with human activities in Akoko environment and also as early colonizers of deforested environment. It is a shrub and often a pioneer colonizer of the Guinean transition woodland, i.e., the transition between the rainforest and the wooded grassland. The human impact on the tropical rainforest probably fostered the growth of Alchornea cordifolia and the presence of its pollen reflects the impact of anthropogenic activities on the study environment. The plant is among the pioneer secondary forest taxa and is common in relict forests, humid vegetations and in thick riparian vegetation along the course of lakes and rivers. It is a copious pollen producer, releasing large quantities of its pollen during the flowering season. The high record of its pollen grains is attributed partly to the anemophilous mode of pollination and the buoyant capacity of the pollen grains [5] [12].

\subsection{Airborne pollen assemblage and its vegetation}

The airborne pollen assemblage trapped in this study generally reflects the regional vegetation of the catchment area which depicts tropical rainforest type. The pollen types recorded in this study represent the subtype vegetation of the study area which includes tropical rainforest, closed/ open forest, riparian forest, woodland shrub grasslands, and human impact ecological zones. The floristic composition of these subtype vegetations are subsumed into three major phyto-ecological groups upon which other small localized subtype vegetations are represented [39]. The site to site variation in number of pollen types portrays not only the existence of subtype vegetations but also the floristic heterogeneity of the vegetation of the study area.

Table 5 Cumulative pollen grains abundance for the phyto-ecological indicator species

\begin{tabular}{llll}
\hline S/N & Phyto-ecological Taxa & Total Pollen Count & Percentage Composition \\
\hline 1 & Lowland Rainforest & 3,651 & $7.21 \%$ \\
2 & Open Forest & 11,503 & $22.72 \%$ \\
3 & Savanna & 28,218 & $55.74 \%$ \\
4 & Human Impact & 7,250 & $14.32 \%$ \\
& Indeterminata & 39 & 0 \\
\hline
\end{tabular}

Of importance also is the presence of pollen types that are indicators of human impact such as those of horticulture (for example, in schools, health centres, streets, etc.), erosion control, etc. which reflected evidence of agriculture and other anthropogenic activities. However, the result of the study was limited in terms of pollen-vegetation representation relationship. The first major reason is concerned with the differences in pollen production and dispersal among tropical plants in our study area [40 - 42]. The entomophilous taxa are known to produce low quantities of pollen grains with poor dispersal quality and the anemophilous taxa are copious pollen producers with good aerodynamic qualities. This advantage makes the anemophilous plants to be well represented or even to be over-represented in pollen assemblage compared to the entomophilous taxa. A second possible explanation is the difficulty of identification of all pollen grains 
to the generic or specific level. For instance, some pollen types are identified only to family level such as Amaranthaceae/ Chenopodiaceae, Cyperaceae and Poaceae.

However, despite these limitations, the identified pollen grains so far still confirm the great floristic diversity and heterogeneity of the vegetation subtypes of the study area and the floristic heterogeneity in the study locations. Similar findings have been reported in Southern Cameroon [42] and South Congo [43].

\subsection{The present vegetation status of Akoko environment}

The airborne pollen assemblages of Akoko environment reflected the flora of the vegetation in flower from October 2016 to September, 2017. The flora grows vegetatively from May to August and flower in September to December, achieving maximum anthesis in November to late December. High level of anthropogenic activities on the environment such as indiscriminate deforestation, annual bush fire and burning of fossil fuel as a result of rapid urbanization has resulted in the unusual increase in the atmospheric heat balance whose implication is the excessive sensible heat experienced in Akoko environment recently. Long term effect could result to climatic change.

The abundance of charred plant particles and smoke from indiscriminate bush fire have not only pollute the environment but has also increase the concentration of greenhouse gases (carbon (iv) oxide, carbon monoxide, chlorofluorocarbon) in the atmosphere whose long term effect could result to global warming.

The activity of cattle herdsmen (overgrazing) has not only destroyed several flora in the vegetation, but has also exposed the topsoil to various agents of denudation making the environment susceptible to desertification. The activities of people that engaged in hunting expedition with the use of bush fire has destroyed several flora and fauna in the environment, these could result to extinction of biodiversity if appropriate conservation and restoration measures are not applied.

\section{Conclusion}

The analysis of the atmospheric pollen content of Akoko environment revealed great diversity of palynomorphs consisting of pollen grains and spores among others. The abundance and persistent release of Elaeis guineensis pollen grain is related to a large extent, the presence of oil palm farm (wild and cultivated) in and around the study environment. The pollen types documented in the study represent the flora of the regional vegetation of the study area which depicts tropical rainforest. The abundance of anemophilous pollen types in the atmosphere is enhanced by their relatively small size and good aerodynamic properties. In contrast, the entomophilous pollen types are large in size and require strong wind velocity and speed to dislodge them. As a result, they are less represented in the atmospheric air. The dominance of pollen produced by Lowland Rainforest and Open Forest taxa, Savanna and Human Impact taxa characteristic of Akoko vegetation, reveals their source area as being mainly from Akoko environment. Pollen assemblages confirmed the vegetation of the study area to be Tropical Rainforest vegetation type despite high level of anthropogenic activities on the environment.

\section{Compliance with ethical standards}

\section{Acknowledgments}

The Corresponding author (Dr. Benjamin Christopher Essien) is grateful to God for wisdom, inspiration and resources and to the Acting Vice Chancellor of Adekunle Ajasin University, Akungba-Akoko; Professor Olugbenga E. Ige (Professor of Palynology and Palaeoecology) for his deep interest, scrutiny and painstaking/ invaluable commitment to this research. I appreciate my wife's (Mrs. Glory) support and understanding during my many months of absence from home.

\section{Disclosure of conflict of interest}

The author declares that there is no conflict of interest.

\section{References}

[1] Ige OE and Essien BC. (2019a). Monitoring environmental change in Akoko North-East Local Government Area of Ondo State, Nigeria through pollen analysis. A paper presented at the $10^{\text {th }}$ Palynological Association of Nigeria (PAN) Conference. Adekunle Ajasin University, Akungba-Akoko, Ondo State, Nigeria. $5^{\text {th }}$ to $9^{\text {th }}$ May. 
[2] Sowunmi MA. (1987). Palynological studies in the Niger Delta. In: E.J. Alagoa, F.N. Anozie and Nwanna Nzewunwa. (Eds.). The early history of the Niger Delta. Helmat Buske Verlag Hamburg. Pp 29-59.

[3] Essien BC and Nkang AE. (2013). Evaluation of the phytoecological indicator species in the airborne palynomorphs from Anyigba, Kogi State, Nigeria. Standard Scientific Research and Essays, 1(14), $398-402$.

[4] Ige OE. (2017). Wanted dead and alive: palynomorphs as microscopic signal to the past and present. $11^{\text {th }}$ inaugural lecture, Adekunle Ajasin University, Akungba-Akoko, Ondo State, Nigeria, 74.

[5] Essien BC. (2019). The Study of Quaternary Airborne Palynomorphs in Akoko Environment, Ondo State, Nigeria. PhD Thesis, Department of Plant Science and Biotechnology, Adekunle Ajasin University, Akungba- Akoko, Ondo State, Nigeria, 487.

[6] Ige OE and Essien BC. (2019b). The applications of pollen analysis in environmental monitoring in Akoko NorthEast Local Government Area of Ondo State, Nigeria. GSC Biological and Pharmaceutical Sciences, 8(1), 64-77.

[7] Roberts N. (1989). The Holocene: an environmental history. Basil-Blackwell. New York, 361.

[8] Davies MB. (1969). Palynology and environmental history during the Quaternary period. American Scientist, 57(3), 317-332.

[9] Overpeck JT. (1985). Quantitative interpretation of fossil spectra: dissimilarity coefficient and the methods of modern analogs. Quaternary Research, 23, 87-108.

[10] Essien BC, Ige OE and Ekeyi D. (2016). Recent pollen rain in Anyigba: an indicator of the vegetation of Kogi State, Nigeria. World Journal of Biology and Medical Sciences, 3(1), 82 - 92.

[11] Agwu COC. (1997). Modern pollen rain in Nsukka: An indicator of the vegetation of Nsukka Plateau. Wurzburger Geographische Arbeiten, 92, 97-115.

[12] Essien BC and Agwu COC. (2013). Aeropalynological study of Anyigba, Kogi State, Nigeria. Standard Scientific Research Essays, 1(13), 347-351.

[13] Agwu COC. (2001). A Study of Niger Delta Environment through air-borne palynomorphs, Port-Harcourt, Nigeria. Palaeoecology of Africa, 27, 191-205.

[14] Moore PD and Webb JA. (1978). An illustrated guide to pollen analysis, First edition. Hodder and Stoughton, KentLondon, 133.

[15] Patrick TM, Kershaw AP and Grindrod J. (2005). Pollen transport and deposition in riverine and marine environments within the humid tropics of North-Eastern Australia. Review of Palaeobotany \& Palynology, 134(12), 55 - 69 .

[16] Njokuocha RC and Agwu COC. (2007). Airborne fungal spores in Nsukka municipality. Nigerian Journal of Botany, 20(2), 349-359.

[17] Davies AL and Bunting MJ. (2010). Applications of palaeoecology in conservation. The Open Ecology Journal, 3, $54-67$.

[18] White F. (1983). The Vegetation of Africa. UNESCO, Paris.

[19] Patton MQ. (1990). Quantitative evaluation and research methods, Second edition. Sage Publications, Newbury Park, CA, 1 - 523.

[20] Tauber H. (1974). A static non-overload pollen collector. New Phytology, 73, 359 - 369.

[21] Tauber H. (1977). Investigations of aerial pollen transport in a forested area. Dansk Botanisk Arkiv, 32(1), 1 121.

[22] Pardoe HS, Giesecke T, van der Knaap WO, Svitavaska-Svobodova H, Kvavadze EV, Panajiotiotidis S, Gerasimidis A, Pidek IA, Zimney M, Swieta-Musznicka J, Latalowa M, Noryskiewiez AM, Bozilova E, Tonkov S, FilipovaMarinova MV, van Leeuwen JFN and Kalnipa L. (2010). Comparing pollen spectra from modified Tauber traps and moss samples: example from a selection of woodlands across Europe. Vegetational History and Archaeobotany, 19, 271 - 283.

[23] Giesecke T, Fontana SL, van der Knaap WO, Pardoe HS and Pidek IA. (2010). From early pollen trapping experiments to the pollen monitoring programme. Vegetational History and Archaeobotany, 19, 247-258.

[24] Erdtman G. (1969). Handbook of Palynology. Muntisguard, Copenhagen, 280 - 285. 
[25] Agwu COC and Akanbi TO (1985). A palynological study of honey from four vegetation zones of Nigeria. Pollen et Spores, 27, 335-348.

[26] Bonnefille R and Riollet G (1980). Pollen des savanna d'Afrique orientale. Anatole Press, Paris, France, 1- 140.

[27] Sowunmi MA. (1995). Pollen of Nigerian plants II. Grana, 34, 120 -141.

[28] Shubharani R, Roopa P and Sivaram V. (2013). Pollen morphology of selected bee forage plants. Global Journal of Bio-Science and Biotechnology, 2(1), 82- 90.

[29] Njokuocha RC. (2006). Airborne pollen grains in Nsukka, Nigeria. Grana, 45, 73-80.

[30] Essien BC. (2014). A comparative analytical study of airborne pollen grains, spores and other palynomorphs within Grimard Catholic hospital, Anyigba, Kogi State, Nigeria. Scholarly Journal of Scientific Research and Essay, 3(5), 56 - 60.

[31] Adeniyi TA, Adeonipekun PA and Olowokedujo JD. (2018). Comparative study of aeropollen and pollinosis cases. Environmental Monitoring and Assessment, 190, 562 -572.

[32] Mabberley DJ. (1987). The plant book. Cambridge University Press, Cambridge.

[33] Latorre F and Belmonte J. (2004). Temporal and spatial distribution of atmospheric Poaceae pollen in Catalonia (NE Spain) in 1996-2001. Grana, 43, 156-163.

[34] McDonald MS. (1980). Correlation of air-borne grass pollen levels with meteorological data. Grana, 19, 53-56.

[35] Singh AB and Babu CR. (1980). Grass pollen content of the atmosphere in Delhi area, India. Grana, 19, 63-65.

[36] Singh AB. (1987). Airborne pollen types of allergic in India. Experimentia Supplementum, 51, 61-68.

[37] Usman SS. (1990). Ecological studies of Opi Lake savanna woodland. Ph.D. Thesis, Department of Botany, University of Nigeria, Nsukka, 202.

[38] Mishra RP, Singh B and Oommachan M. (2002). Airborne pollen flora of Jabulur-the Central India. Aerobiologia, $18,73-81$.

[39] Essien BC, Idachaba SO and Okai EE. (2019). Index of the phytoecological indicator species in the prevalent airborne pollen types in Akoko environment, Ondo State, Nigeria. GSC Biological and Pharmaceutical Sciences, 8(3), 23-41.

[40] Bonnefille R, Buchet G, Friis G, Kelbessa E and Mohammed U. (1993). Modern pollen rain on altitudinal range of forests and woodlands in South-West Ethiopia. Opera Botanica, 121, 71-84.

[41] Vincens A, Ssemmanda I, Roux M and Jolly D. (1997). Study of the modern pollen rain in Western Uganda with a numerical approach. Review of Palaeobotany and Palynology, 96, 145-168.

[42] Vincens A, Dubois MA, Guillet B, Achoundong G, Buchet G, Kamgang Kabeyene Bayala V, de Namur C and Riera B. (2000). Pollen rain-vegetation relationships along a forest-savanna transect in southeastern Cameroon. Review of Palaeobotany and Palynology, 110, 191-208.

[43] Elenga H, Nammur de C, Vincens A, Roux M and Schwartz, D. (2000). Use of plots to define pollen-vegetation relationship in densely forested ecosystems of Tropical Africa. Review of Palaeobotany and Palynology, 112, 7696.

\section{How to cite this article}

Essien BC and Ige OE. (2020). Recent pollen rain analysis in Akoko environment as indicator of the vegetation of Ondo State, Nigeria. GSC Biological and Pharmaceutical Sciences, 12(1), 116-137. 